\title{
Rapid Molecular Diagnosis of Parechovirus Infection Using the Reverse Transcription Loop- Mediated Isothermal Amplification Technique
}

Tadafumi Yokoyama ( $\square$ tadafumiy@staff.kanazawa-u.ac.jp )

Kanazawa University

Yuko Tasaki

Kanazawa University

Natsumi Inoue

Kanazawa University

Naotoshi Sugimoto

Kanazawa University

\section{Eri Nariai}

Ishikawa Prefectural Institute of Public Health and Environmental Science

\section{Sanae Kuramoto}

Ishikawa Prefectural Institute of Public Health and Environmental Science

\section{Taizo Wada}

Kanazawa University

\section{Research Article}

Keywords: HPeV, RNAs, Human, Parechovirus

Posted Date: January 13th, 2021

DOl: https://doi.org/10.21203/rs.3.rs-137528/v1

License: (c) (i) This work is licensed under a Creative Commons Attribution 4.0 International License. Read Full License

Version of Record: A version of this preprint was published at PLOS ONE on November 29th, 2021. See the published version at https://doi.org/10.1371/journal.pone.0260348. 


\section{Abstract}

Objectives Human parechovirus (HPeV), especially HPeV A3 (HPeV3), causes sepsis-like diseases and sudden infant death syndrome in neonates and young infants. However, commercial laboratory tests for HPeVs, including HPeV3, do not exist.

Methods We originally designed inner primers, outer primers, and loop-primers on the $5^{\prime}$ untranslated region of HPeV3. We used HPeV3 ribonucleic acids (RNAs), other viral RNAs, and clinical stool samples to confirm whether our designed primers would allow the detection of HPeV3 with the reverse transcription loop-mediated isothermal amplification (RT-LAMP) technique.

Results We created three combinations of primers and confirmed that all primer sets allowed the detection of HPeV3 RNAs in only 60 minutes. These primer sets had cross-reactivity with HPeV type 1 (HPeV1), but all sets showed negative results when we applied Coxsackievirus, Echovirus, Enterovirus, Norovirus, and Adenovirus genomes. Four of six stool samples, which were obtained from newborn and infant patients with sepsis-like symptoms, showed positive results with our RT-LAMP technique.

Conclusions This report is the first showing that HPeVs can be detected with RT-LAMP. Based on this finding, HPeV infection can be diagnosed faster, more easily, and with less cost. This technique is clinically useful for newborn and infants who have sepsis-like symptoms.

\section{Introduction}

Human parechovirus $(\mathrm{HPeV})$ is increasingly being recognized as a potentially severe viral infection in neonates and young infants [1-3]. HPeVs usually cause the "common cold" (i.e., respiratory or gastrointestinal illness similar to rhinovirus, enterovirus, and coronavirus) [1-3]. However, HPeVs have been implicated in cases of neonatal sepsis-like diseases, encephalitis, sudden infant death syndrome, and myocarditis [1-3]. HPeVs belong to the family Picornaviridae and are divided into 19 genotypes [1].

Human parechovirus A3 (HPeV3) is the most clinically important genotype among HPeVs because of its association with severe disease in newborns and young infants [1, 3]. HPeV3-infected infants can present with a sepsis-like symptom, often with central nervous system involvement, which is difficult to differentiate clinically from bacterial sepsis $[1,3]$. They may present with fever, tachycardia, and erythema [1]. Abdominal distension and navel protrusion are also seen in these patients [1]. Characteristic laboratory findings are cytopenia and elevated lactate dehydrogenase and ferritin levels, which suggest hemophagocytic lymphohistiocytosis [1]. Seizures or significant neurological impairment sometimes occur [1]. Severe HPeV3 infection in infants is also associated with a risk of long-term complications [1].

The application of molecular diagnostic methods enables the early recognition of HPeV infections. Early recognition is important because it may reduce the use of antibiotics and shorten the duration of hospital admissions for patients with mild to moderate disease. Early diagnosis is also likely to lead to appropriate investigations and follow-up for potential complications in infants who are severely affected. 
However, commercial laboratory tests do not exist for HPeVs, including HPeV3. Only research institutes can detect HPeVs by using nested polymerase chain reaction (PCR) and direct sequencing of amplified PCR products [1-3].

We developed an easy and faster molecular diagnostic method to detect HPeV by using the reverse transcription loop-mediated isothermal amplification (RT-LAMP) technique. When using the RT-LAMP technique that we developed, we were able to diagnose HPeV in 2 hours in small steps.

\section{Results}

\section{Development of RT-LAMP method for HPeVs}

The $5^{\prime}$ UTR is $700 \mathrm{bp}$ in length. When we attempted to find combinations of primers using whole $700 \mathrm{bp}$ bases of the 5' UTR by using PrimerExplorer V5 (Eiken Chemical Co., Ltd.), we could not find any appropriate combinations of primers. Therefore, while shifting the range (e.g., 1-400, 11-410, 21-340, ..., 301-700 (i.e., the last 5' UTR), we comprehensively extracted all combinations of primers (i.e., FIP, BIP, F3, and B3). The results showed eight candidate combinations (Supplemental Table 2). From these combinations, we chose three combinations (Set $\mathrm{A}^{\text {loop-; }} \mathrm{B}^{\text {loop- }}$, and $\mathrm{C}^{\text {loop }}$ ) because they were most frequently duplicated (Figure 1-a and Table). Sets $\mathrm{A}^{\text {loop- }} \mathrm{B}^{\text {loop- }}$, and $\mathrm{C}^{\text {loop- }}$ - were extracted 12 times in our experiments, but the other five combinations of the primers were extracted only once or twice.

When we used HPeV3 viral RNAs for RT-LAMP, Sets $\mathrm{A}^{\text {loop- }}, \mathrm{B}^{\text {loop- }}$, and $\mathrm{C}^{\text {loop- }}$ showed positive results. However, we tested multiple times and these sets had unstable or unreproducible rates (positive rate, \%; [i.e., number of positive samples/number of tested samples]), as follows: Set $A^{\text {loop }}$; $33.3 \%(6 / 18)$; Set $B^{\text {loop- }}, 44.4 \%(8 / 18)$, and Set $C^{\text {loop- }}, 86.0 \%(6 / 7)$.

Therefore, we added two more primers, loop- $F$ and loop- $\mathrm{R}$, to increase the sensitivity of the RT-LAMP method. We automatically designed loop-R by using PrimerExplorer V5 (Eiken Chemical Co., Ltd.), but we manually designed loop- $F$ because PrimerExplorer V5 could not answer the loop- $F$ candidate (Figure 1-a and Table). As a result, we could detect HPeV3 by using primer Set A (Set $A^{\text {loop- }}+$ loop-F/R), Set B (Set $B^{\text {loop- }}+$ loop-F/R), and Set C (Set $C^{\text {loop- }}+$ loop-F/R) (Figure 1-b). The RT-LAMP products that included the target sequence and the reverse complementary sequence were confirmed by direct sequencing (data not shown).

\section{Minimum reaction time and amount of viral RNAs}

We tested RT-LAMP by changing the reaction times to 20 minutes, 30 minutes, 40 minutes, ..., 110 minutes. We also tested RT-LAMP by applying $1.18 \times 10^{2} \mathrm{ng}, 1.18 \times 10 \mathrm{ng}, 1.18 \mathrm{ng}, \ldots ., 1.18 \times 10^{-5} \mathrm{ng}$ of total viral RNAs. The results are shown in Supplemental Table 3. When we applied more than $1.18 \times 10^{-1} \mathrm{ng}$ of the total viral RNAs, positive results were visible at 20 minutes in Set A and Set B. Set C showed optically positive results at 30 minutes. However, when we applied less than $1.18 \times 10^{-1} \mathrm{ng}$ of the total viral RNAs, 
the reaction became unstable. Only Set $\mathrm{C}$ showed positive results at 50 minutes for all amounts of viral RNAs. Therefore, we confirmed that our RT-LAMP technique could detect at least $118 \mathrm{pg}$ of the total viral RNAs in stool samples and could show positive results in 30 minutes.

We also changed the reaction temperature, using $59^{\circ} \mathrm{C}, 61^{\circ} \mathrm{C}, 63^{\circ} \mathrm{C}, 65^{\circ} \mathrm{C}$, and $67^{\circ} \mathrm{C}$. The most appropriate and stable temperature was $63^{\circ} \mathrm{C}$ (data not shown).

\section{Specificity of the RT-LAMP method}

We conducted RT-LAMP using samples that were already known the etiology (two more HPeV3, one HPeV1, one Coxsackievirus B5, one Echovirus 11, one Enterovirus D68, one Enterovirus A71, three Noro, two Adeno. HPeV3 and HPeV1 showed positive results in our RT-LAMP assay. However, all other samples were negative (Figure 2 and Supplemental Table 4).

\section{RT-LAMP analysis using clinical samples}

The stools of six anonymous febrile infants were obtained at the time of suspected HPeV infection. From these stools, we extracted viral RNAs. The concentration of viral RNA solutions was $84.8 \pm 25.2 \mu \mathrm{g} / \mathrm{mL}$ (expressed as the mean \pm standard deviation).

We applied $2 \mu \mathrm{L}$ of viral RNA solution, which was $169.7 \pm 50.4 \mathrm{ng}$ of viral RNAs, for the RT-LAMP experiments. The LAMP products were visible in the stool samples of four patients (Patients \#3-\#6) and the positive control (Figure 3). However, no RT-LAMP products were visible in the negative control and in Patients \#1 and \#2. RT-LAMP experiments were conducted using both Sets A, B, and C, and all showed the same results (Supplemental Figure).

In the six stools, we simultaneously tried to detect HPeVs genomes by using the nested PCR technique. We detected HPeV3 in four samples and did not detect HPeVs in two samples. This finding corresponded to the RT-LAMP results. An important finding was that we could know the results in 2 hours after collecting stool samples when using the RT-LAMP technique, but in 2 days when using the nested PCR technique. In addition, all RT-LAMP products were confirmed by direct sequencing (data not shown).

\section{Discussion}

This study was the first to detect HPeVs by using the RT-LAMP method.

The PCR method is available to diagnose HPeVs infection. The PCR test requires specialized expertise and is available at limited clinical laboratories. In addition, it requires several steps, which take time to accomplish. These disadvantages of PCR tests are problematic for newborns and infants whose physical condition varies hourly. Therefore, we believe that a rapid diagnostic system to detect HPeVs may be clinically useful for these patients. 
The LAMP reaction, an alternative nucleic acid amplification method developed by Notomi et al. [4], is based on strand displacement by DNA polymerase under isothermal conditions in which the temperature range is $60^{\circ} \mathrm{C}-65^{\circ} \mathrm{C}[4-7]$. The LAMP assay is very specific, compared to other molecular detection methods, because four primers are necessary that recognize six specific regions of the target gene for amplification [4-7]. The method generates a large amount of amplification products in positive samples ( $10^{9}$ - to $10^{10}$-fold in $15-60$ minutes), thereby allowing some assessment of these products with the naked eye and fluorescent dye [4-7]. Another advantage of the LAMP method is that amplification occurs at one temperature; therefore, it does not require a thermal cycler [4-7]. The RT-LAMP assay, which is carried out with a RT step and a LAMP reaction in a single tube, is a simple, highly specific, rapid, and cost-effective method $[6,7]$. Fewer operating steps in RT-LAMP is also advantageous in preventing contamination, and thus being safe for technicians.

In this study, we have developed an RT-LAMP assay for HPeVs detection by designing specific primers, based on the 5' UTR region of the HPeV3 viral gene. We compared in silico the primer alignment to the $5^{\prime}$ UTR region of other types of HPeVs by using Basic Local Alignment Search Tool (National Center for Biotechnology Information, Bethesda, MD, USA). As a result, our primers showed some homology in HPeV1, HPeV4, HPeV5, HPeV8, HPeV14, and HPeV18 (data not shown). Therefore, our RT-LAMP assay may have cross-reactivity to the other HPeVs, except for HPeV3. We indeed confirmed that HPeV1 was also detected in our primer sets.

Aside from HPeV3, HPeV infections are common around the world and have been identified on every inhabited continent [2]. However, an accurate prevalence rate is unknown and varies, depending on the age of the individuals included in the study population and the sampling sites chosen [2]. HPeV A1 (HPeV1) is the most prevalent genotype and most commonly causes gastrointestinal and respiratory diseases [1, 2]. The symptoms are usually mild, although HPeV1 sometimes causes respiratory disease in young children, and initially presents as coryza, accompanied by cough and dyspnea. Occasional HPeV1 cases present with necrotizing enterocolitis, encephalitis, and Reye's syndrome [1, 2]. HPeV1 outbreaks sometimes occur in nursery schools and neonatal intensive care units [1, 2]. Frequency of HPeV1 infection is not uncommon, but unknown because clinicians cannot diagnose $\mathrm{HPeV}$ infection including $\mathrm{HPeV} 1$ and HPeV3 as easy as rapid diagnosis kit (i.e., influenza virus). Clinicians may diagnose a patient as having "some kind of viral infection." In some more severe cases, a research institute can help to diagnose the infection.

Therefore, we believe that our RT-LAMP method withstands criticism because the most important purpose is the detection of HPeVs, regardless of the genotype of HPeVs as a screening. If our RT-LAMP method becomes widespread, clinicians and patients can easily know the answer to "some kind of viral infection." However, further experiments are necessary to design HPeV type-specific primers and develop the appropriate LAMP condition.

We completely detected HPeV3 from clinical stool samples. These patients were all 1-month-old babies and showed sepsis-like symptoms. We successfully diagnosed these patients as having HPeV infection 
in 2 hours. However, if we had used the nested PCR technique, we would have known the results in 2 days. This delay in determining a diagnosis is sometimes critical for infants and newborns. Our RT-LAMP technique may solve this problem.

In addition, $\mathrm{HPeV} 3$ infection is usually asymptomatic or manifests as a faint common cold in older children and adults. HPeV3 is known to cause myalgia/myositis in all ages and is recognized as an important pathogen for all ages [1]. Our RT-LAMP method may also be useful for myalgia/myositis in all ages.

In our experiment, we clarified that $50-100 \mu \mathrm{g} / \mathrm{mL}$ of total viral RNAs can be collected from $50-100 \mu \mathrm{L}$ of stools. We also confirmed that $100 \mathrm{pg}$ to $500 \mathrm{ng}$ of total viral RNAs were sufficient to conduct our RTLAMP technique and obtain accurate results. We usually applied 2-5 $\mu \mathrm{L}$ of viral RNA solutions; thus, we applied $100-200 \mathrm{ng}$ of total viral RNAs. Therefore, $50-100 \mu \mathrm{L}$ of stool is sufficient.

On account of the limited number of cases, we were unable to determine (1) the sensitivity and specificity of each primer set and (2) the usefulness of RT-LAMP, even if serum, urine, and cerebrospinal fluids samples were applied. A large prospective study is necessary to confirm these limitations.

In conclusion, by using our RT-LAMP technique for HPeVs, an HPeV infection can be diagnosed faster, easily, and at less cost. It is clinically useful for all patients with gastrointestinal and respiratory diseases, myalgia/myositis, encephalitis, etc., especially for newborn and infants who have sepsis-like symptoms.

\section{Materials And Methods}

\section{Design of the RT-LAMP primers}

The sequence of HPeV3 was downloaded from the National Center for Biotechnology Information (Bethesda, MD, USA). Based on the highly conserved 5' untranslated region (UTR) sequence of HPeV3, we originally designed inner primers (i.e., FIP and BIP), outer primers (i.e., F3 and B3), and loop-primers (loopF and loop-R) on the 5' UTR of HPeV3 by using PrimerExplorer V5 (Eiken Chemical Co., Ltd., Taito-ku, Tokyo, Japan).

\section{RT-LAMP assay}

HPeV3 viral ribonucleic acid (RNA), which was the positive control, was the kind gift of the Health and Food Safety Department at Ishikawa Prefectural Institute of Public Health and Environmental Science (Kanazawa, Ishikawa, Japan). For the detection of viral RNA by LAMP assay, we used the Loopamp RNA Amplification Kit (Eiken Chemical Co.) and followed the manufacturer's protocol. In brief, we incubated viral RNA with enzymes, buffers, deoxyribose nucleoside triphosphates, and primers at $63^{\circ} \mathrm{C}$ for 1 hour. The enzymes were thereafter deactivated at $80^{\circ} \mathrm{C}$ for 5 minutes. The products obtained by RT-LAMP were detected visually. The RT-LAMP products were confirmed by direct sequencing using other originally designed primers (Supplemental Table 1). 


\section{Direct sequencing of HPeV gene amplified with nested PCR and RT-LAMP}

Complementary DNA (cDNA) was synthesized with reverse RT using Superscript II Reverse Transcriptase (Invitrogen, Carlsbad, CA, USA). The samples were first screened with nested PCR targeting the partial $5^{\prime}$ UTR of HPeVs. The test-positive samples were assayed using nested PCR targeting the VP1 region for genotyping [2,3]. Amplified DNA was directly sequenced using the BigDye Terminator Cycle Sequencing Kit (Thermo Fisher Scientific, Waltham, MA, USA) in both directions. Each genotype was determined by comparing the nucleotide sequence with the available HPeV sequences from GenBank using the Genetyx program (GENETYX, Shibuya-ku, Tokyo, Japan). For RT-LAMP products, as for nested PCR, we directly sequenced the amplified DNA with the BigDye Terminator cycle sequencing kit (Thermo Fisher Scientific) in both directions by using primers for sequencing (Supplementary Table 1).

\section{Sensitivity and specificity analysis of HPeV RT-LAMP}

We cultured virus-infected cells and harvested two more HPeV3, one HPeV1, one Coxsackievirus B5, one Echovirus 11, one Enterovirus D68, and one Enterovirus A71, and one Adenovirus (Adeno). In addition, we collected the stools of three patients with Norovirus (Noro) gastroenteritis and one patient with Adeno gastroenteritis and extracted Noro RNAs and Adeno DNA from the samples. All samples were tested for HPeVs by using RT-LAMP.

\section{RT-LAMP analysis using clinical samples}

The stools of six anonymous febrile infants were obtained at the time of the suspicion of HPeV infection, after obtaining informed consent from the parents. Fifty to $100 \mu \mathrm{L}$ of stools were diluted with $200 \mu \mathrm{L}$ of distilled water and vortexed strongly for 5 minutes. The samples were then centrifuged at 15,000 rpm for 5 minutes at $4^{\circ} \mathrm{C}$. One hundred forty microliters of the supernatants were applied for the extraction of viral RNA. Viral RNA was extracted from supernatants by using QIAmp Viral RNA Mini Kit (Qiagen, Hilden, Germany), based on the manufacturer's instructions. Extracted viral RNA was stored at $-80^{\circ} \mathrm{C}$ until amplification using RT-LAMP and nested PCR detection.

\section{Ethics}

This study was approved by the institutional review board of Graduate School of Medical Sciences at Kanazawa University [Kanazawa, Japan; protocol number: 2014-072(1686)]. This study was conducted according to the Declaration of Helsinki. All experiments were performed in accordance with relevant guidelines and regulations (including informed consent from all participants and parents).

\section{Declarations}

\section{Author Contributions}

Substantial contributions to conception and design, acquisition of data, or analysis and interpretation of data; Tadafumi Yokoyama, Yuko Tasaki, Natsumi Inoue, Naotoshi Sugimoto, Eri Nariai, Sanae Kuramoto, 
Taizo Wada

Drafting the article or revising it critically for important intellectual content; Tadafumi Yokoyama, Naotoshi Sugimoto, Taizo Wada

Final approval of the version to be published; Tadafumi Yokoyama, Taizo Wada

\section{Disclosure of Funding}

This study was supported by Grants-in-Aid for Science and Culture from the Ministry of Education, Culture, Sports, Science, and Technology of Japan. This research did not receive any specific grant from funding agencies in the public, commercial, or not-for-profit sectors.

\section{Conflict of Interest}

None.

\section{Consent Statement}

We obtained informed consent from the parents of all patients.

\section{References}

1. Olijve, L., Jennings, L. \& Walls, T. Human Parechovirus: an Increasingly Recognized Cause of SepsisLike Illness in Young Infants. Clinical Microbiology Reviews. 31, e00047-17 (2018).

2. Harvala, H. et al. Epidemiology and clinical associations of human parechovirus respiratory infections. J Clin Microbiol. 46, 3446-3453 (2008).

3. Ito, M. et al. Detection of human parechoviruses from clinical stool samples in Aichi, Japan. J Clin Microbiol. 48, 2683-2688 (2010).

4. Notomi, T. et al. Loop-mediated isothermal amplification of DNA. Nucleic Acids Res. 28, e63 (2000).

5. Zhao, H. B. et al. Development of Loop-Mediated Isothermal Amplification (LAMP) for Universal Detection of Enteroviruses. Indian J Microbiol. 54, 80-86 (2014).

6. Monazah, A., Zeinoddini, M. \& Saeeidinia, A. R. Evaluation of a rapid detection for Coxsackievirus B3 using one-step reverse transcription loop-mediated isothermal amplification (RT-LAMP). J Virol Methods. 246, 27-33 (2017).

7. Baek, Y. H. et al. Simple, rapid and sensitive portable molecular diagnosis of SFTS virus using reverse transcriptional loop-mediated isothermal amplification (RT-LAMP). J Microbiol Biotechnol. 28, 19281936 (2018).

\section{Tables}

Due to technical limitations, table 1 is only available as a download in the Supplemental Files section. 


\section{Figure 1}

\section{(a)}

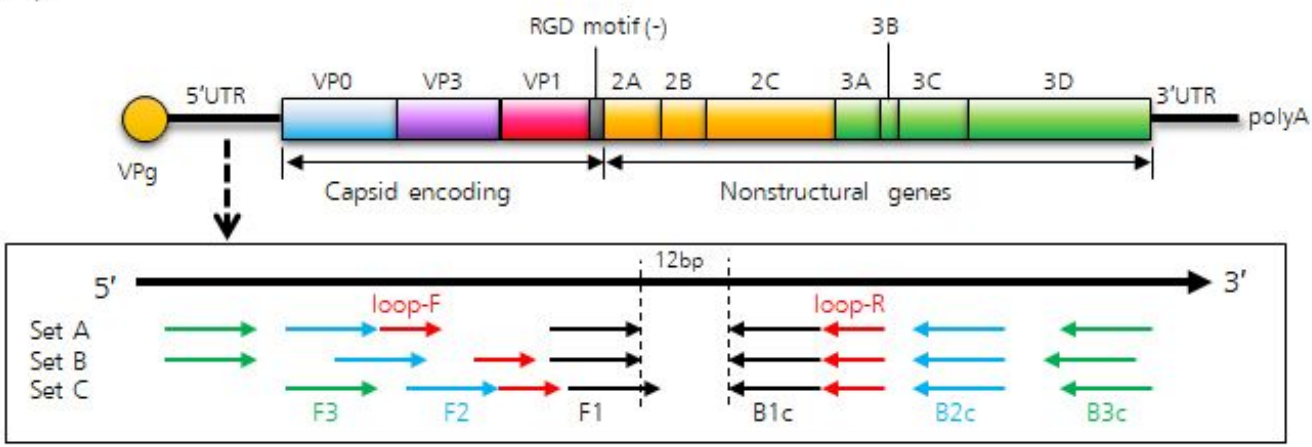

(b)

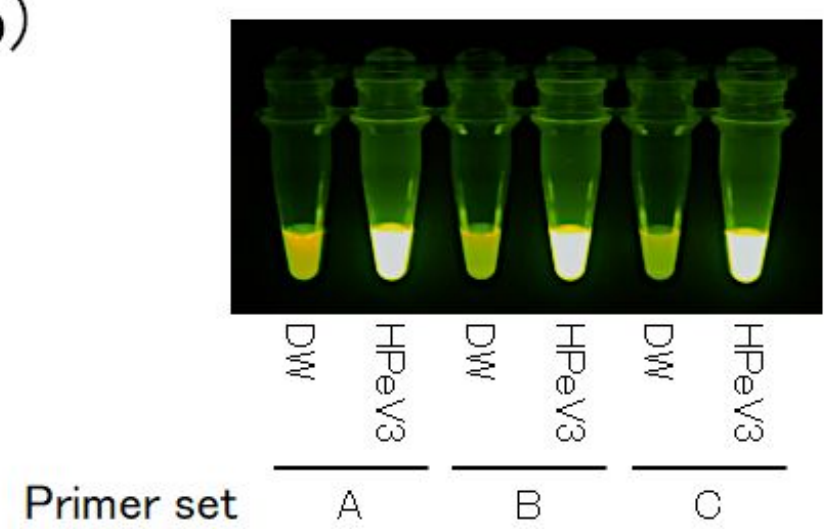

\section{Figure 1}

Development of the RT-LAMP method for HPeVs (a) The HPeV3 genome and scheme of the combinations of the primers for HPeVs. We designed three primer sets for RT-LAMP on the 5' UTR of HPeV3. (b) Results of RT-LAMP using the three primer sets. Tubes containing HPeV3 RNAs show a bright fluorescence, compared to tubes with distilled water. DW, distilled water; $\mathrm{HPeV}$, human parechovirus; HPeV3, human parechovirus A3; RT-LAMP, reverse transcription-loop-mediated isothermal amplification; UTR, untranslated region 


\section{Figure 2}

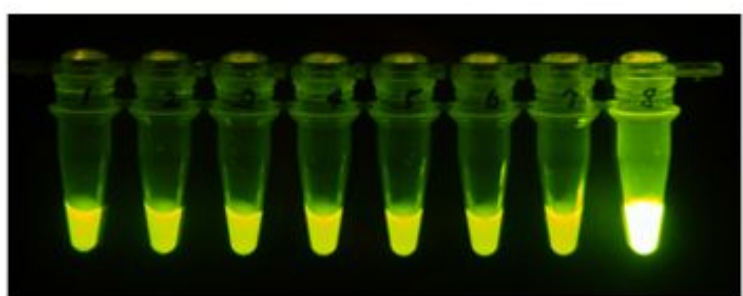

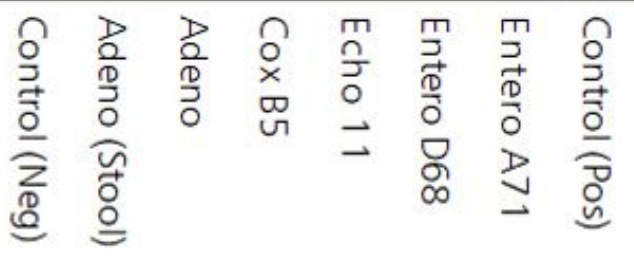

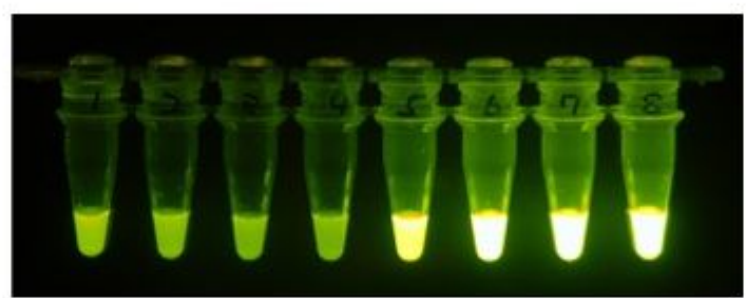

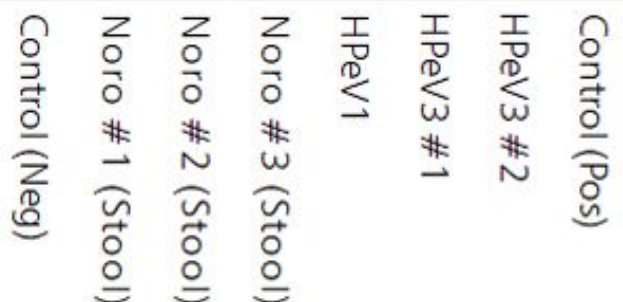

\section{Figure 2}

Results of RT-LAMP using different viral genomes Tubes containing HPeV1 and HPeV3 show positive results, whereas the other tubes show negative results. Cox B5, Coxsackievirus B5; Echo 11, Echovirus 11; Entero A71, Enterovirus A71, Noro, Norovirus; Entero D68, Enterovirus D68; HPeV1, human parechovirus type $1 ; \mathrm{HPeV} 3$, human parechovirus type 3; Neg, negative; Pos, positive; RT-LAMP, reverse transcription loop-mediated isothermal amplification 


\section{Figure 3}

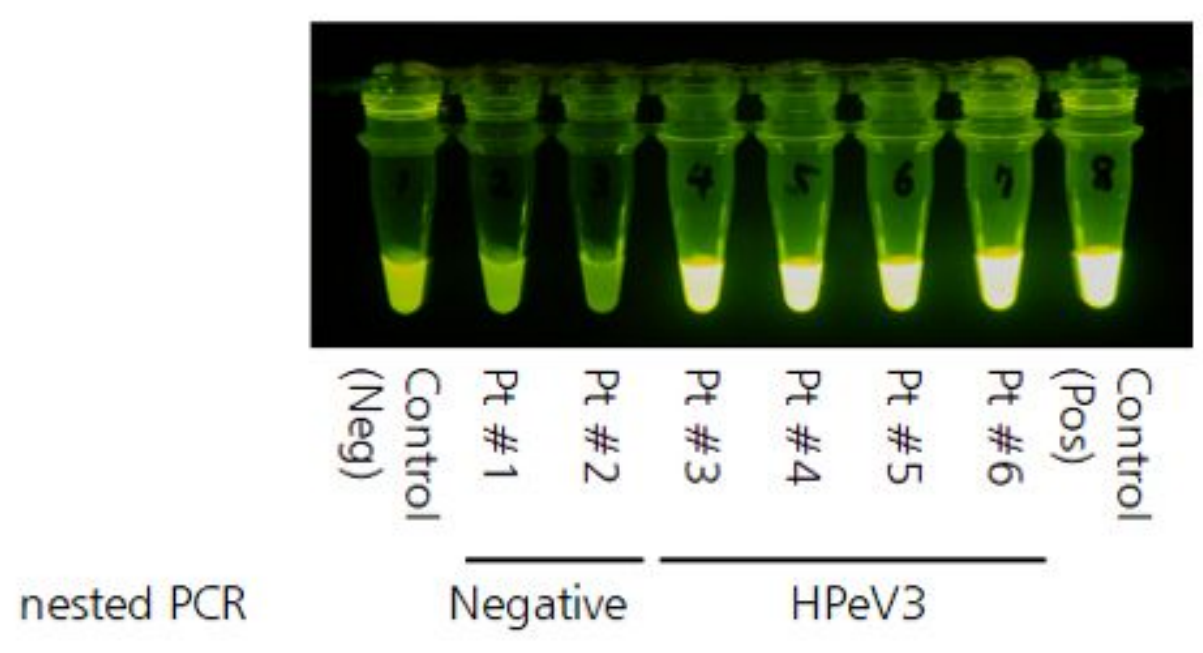

Figure 3

Representative picture of the results of RT-LAMP by using clinical samples The stool samples of two patients (Patient \#1 and Patient \#2) show negative results. The stool samples of four patients (Patients \#3-\#6) show positive results for RT-LAMP. This figure is from primer Set A. The results of nested PCR for HPeV3 completely corresponded to the results of RT-LAMP. HPeV3, human parechovirus A3; Neg, negative; PCR, polymerase chain reaction; Pos, positive; RT-LAMP, reverse transcription loop-mediated isothermal amplification

\section{Supplementary Files}

This is a list of supplementary files associated with this preprint. Click to download.

- Table1.jpg

- SupplementaryFile.docx 